\title{
Atomically Resolved Scanning Confocal Electron Microscopy Using a Double Aberration-corrected Transmission Electron Microscope
}

\author{
Peng Wang ${ }^{1}$, Angus I. Kirkland ${ }^{2}$, Peter D. Nellist ${ }^{2}$, Adrian J. D’Alfonso ${ }^{3}$, Andrew J. Morgan ${ }^{3}$, Leslie J. \\ Allen $^{3}$, Ayako Hashimoto ${ }^{4,5,6}$, Masaki Takeguchi ${ }^{4,6}$, Kazutaka Mitsuishi ${ }^{4,5,6}$ and Masayuki Shimojo ${ }^{7}$ \\ 1. National Laboratory of Solid State Microstructures and College of Engineering and Applied sciences, \\ Nanjing University, Nanjing 210093, People's Republic of China \\ 2. Department of Materials, University of Oxford, Parks Road, Oxford OX1 3PH, UK \\ 3. School of Physics, University of Melbourne, Parkville, Victoria 3010, Australia. \\ 4. Surface Physics and Structure Unit, National Institute for Materials Science, 1-2-1 Sengen, Tsukuba, \\ 305-0047, Japan. \\ 5. Global Research Center for Environment and Energy based on Nanomaterials Science, National \\ Institute for Materials Science, 1-2-1 Sengen, Tsukuba, 305-0047, Japan. \\ 6. Electron Microscopy Station, National Institute for Materials Science, 1-2-1 Sengen, Tsukuba, 305- \\ 0047, Japan. \\ 7. Department of Materials Science and Engineering, Shibaura Institute of Technology, \\ 3-7-5, Toyosu, Koto-ku, Tokyo, 135-8548, Japan
}

The recent development of spherical aberration correctors for both transmission electron microscopy (TEM) and scanning TEM (STEM) has led to a reduced depth of field that can be as small as a few nanometres. An opportunity therefore exists to optically section the sample to provide three-dimensional (3D) information. Optical sectioning experiments have been demonstrated using spherical aberration corrected TEM/STEM instruments operating in a scanning confocal electron microscopy (SCEM) configuration [1]. In the SCEM configuration (shown in Fig. 1 a)), the pre-specimen optics are the same as STEM, whilst the post-specimen optics are used to image electrons that have been scattered from the confocal point onto an aperture in the detector plane, known (taking the name used in the light-optical equivalent) as the pin-hole. Electrons scattered from elsewhere are focused to a point either above or below the pin-hole such that their contribution to the image intensity is reduced.

Based on this optical configuration, various operation modes have been experimentally developed, including bright-field (BF) [2, 3], energy-filtered (EF) [4] and annular dark-field SCEM [5]. Cosgriff et al. [6] and D'Alfonso et al. [7] have theoretically examined the contrast mechanisms for SCEM using elastically and inelastically scattered electrons, respectively. Their work showed that elastic SCEM or BFSCEM image contrast is weak and that dynamical scattering in the form of channelling plays an important role in the contrast mechanisms for thicker samples. However, inelastic SCEM or EFSCEM gives improved depth selectivity, which allows 3-D structure determination. Importantly they showed that the transfer function does not have a missing cone, so that even laterally extended or planar objects can be depth located.

Here we review the developments of all the modes and compare them in terms of their optical setups, imaging contrast mechanisms and applications for different type of materials. Furthermore, we will demonstrate lateral atomic resolution in an EF-SCEM optical sectioning experiment using a silicon sample in the $<110>$ orientation [8] as shown in Fig. 2. We will show the effect of the confocal geometry on the contrast of the atomic resolved image. The experiments and simulations presented here show, however, that the effects of channelling absorption (as shown in Fig. 1b)) and delocalisation are 
still very significant and must be taken into account for the interpretation of atomically resolved EFSCEM imaging [9].

\section{References:}

[1] P.D. Nellist and P. Wang, Annual Review of Materials Research, Annual Reviews 42 (2012), p. 125143.

[2] K. Mitsuishi et al, Ultramicroscopy 111 (2010) p20-26.

[3] P. Wang et al, Ultramicroscopy 111 (2011) p877-886.

[4] P. Wang et al, Physical Review Letters 104 (2010) p200801.

[5] A. Hashimoto et al, Appl. Phys. Lett. 101 (2012) p253108.

[6] E.C. Cosgriff et al, Ultramicroscopy 108 (2008) p1558-1566.

[7] A.J. D'Alfonso et al, Ultramicroscopy 108 (2008) p1567-1578.

[8] P. Wang et al, Ultramicroscopy 134 (2013) p185-192.

[9] Financial support from the Leverhulme Trust (F/08 749/B), the EPSRC (EP/F048009/1) and the ARC's Discovery Projects funding scheme (DP110102228) is gratefully acknowledged.

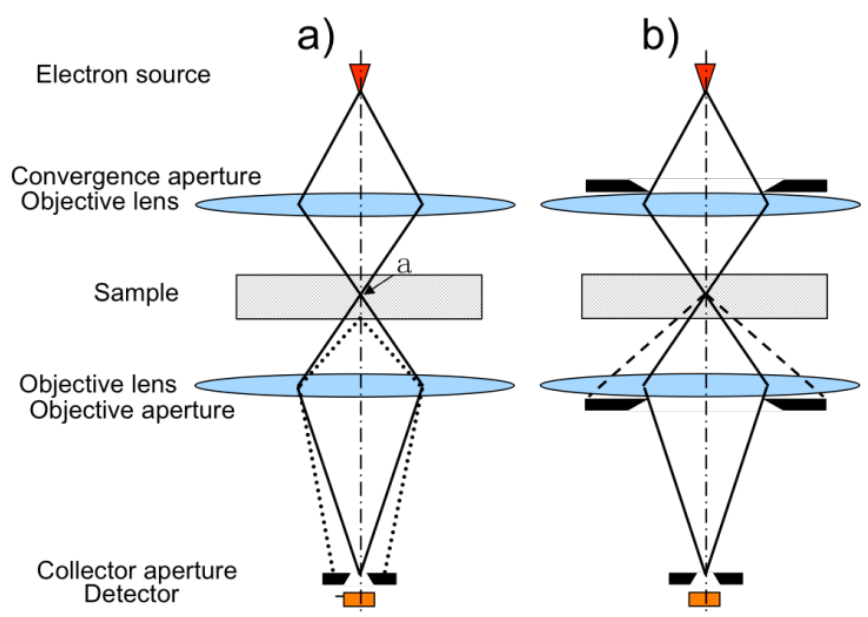

Figure 1. a) Schematic diagram of ray paths for SCEM, showing that beams (---) scattered away from the confocal point, marked a, are rejected by the collector aperture; b) The electrons detected in SCEM imaging can be angularly limited by the objective aperture in the post-specimen optics.
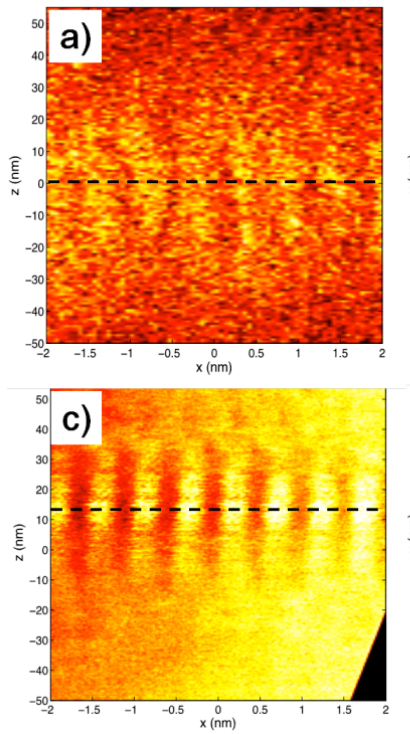
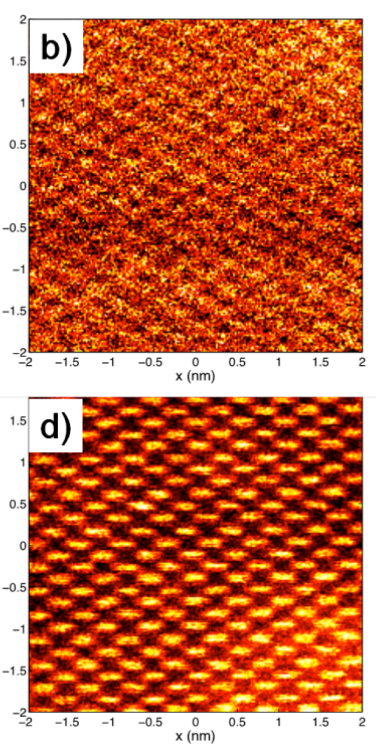

Figure 2. (a) Experimental EFSCEM x-z scan optically sectioned from the $\mathrm{Si}<110>$ slab with a collector aperture corresponding to a diameter of $0.32 \mathrm{~nm}$; (b) EFSCEM $x-y$ scans acquired at the depth indicated by a dashed line in (a); (c) is STEM-HAADF $x-z$ scan and d) is STEM-HAADF $x-y$ scan acquired at the depth indicated by a dashed line in (b). Note that the dashed lines in a) and c) indicate the depth where the maximum signal of the lattice fringes appears in either of them. 